\title{
QUANTUM COMPUTING WILL COMPLETELY CHANGE THE HEALTHCARE INFRASTRUCTURE TO NEW LEVEL AND - HELP TRANSFORM THE HEALTHCARE FROM PREVENTIVE TO PREDICTIVE HEALTHCARE: A FUTURE PERSPECTIVE
}

\section{Devanssh Mehta *}

Director, TRM Writers LLP, Meerut, Uttar Pradesh, India

Mobile: +917351348736; Email: devanshm84@gmail.com

\section{ABSTRACT}

Healthcare across the world is advancing to all new levels because of technological advances. Recent developments like Artificial Intelligence, Big data analysis, and Quantum computing are helping to take the healthcare systems to all new levels. The present report is written in order to put across the theory that Quantum computing will help in revolutionizing healthcare, be it pharma, or diagnostic industry or medical treatment sector. Quantum computing has helped us to leap from bits to qubits means superpower computing infrastructure which will impact the whole healthcare sector.

Keywords: Quantum computing, Qubits, Healthcare, Pharmaceuticals 
The Healthcare sector is widely helping the people in their fight against diseases and ailments. The healthcare workers are helping humanity to overcome the effects of diseases or ailments through newer treatment protocols and medicines. Technological advances happening including the recent ones, such as Quantum computing and Artificial intelligence have also transitioned its effects on the healthcare sector.

Artificial Intelligence which has helped the healthcare workers in fastening the treatment protocols has totally revolutionized the healthcare system. Another newer advancement which is at present in the discussion is Quantum computing. Now, what is Quantum computing?

Quantum computing takes the help of quantum computers which work at higher speeds and calculates the analysis in Qubit form. Qubit is a phenomenon where simultaneous data calculations are carried out in ones and zeros at the same time. Bits calculate the data in either one or zero which is currently powered in present computers. Quantum computers are the advanced computers where they have the ability to do the analysis as well as calculations in space of ones and zeros simultaneously. Now, this means that the present computers which calculate and reads the data in bit form will take the analysis of all the publications in the PubMed database in a thousand years. However, Quantum computers can analyze and interpret the millions of publications in the PubMed database in a single day. That's what Quantum computing is all about.
The significance of Quantum computing to healthcare, pharma sector is that supersonic drug discoveries will happen in reality, that reducing the cost and time period of developing newer drugs. Not only this it can also help in securing the data being interpreted by artificial intelligence through Quantum uncertainty, thus making this technology a leap in human advancement.

Quantum computing will help in the development of super drugs, super transdermal patches, and super diagnostic technologies using the qubit technology.

On the whole Quantum computing will not only advance the preventive healthcare but also transform the healthcare to the predictive atmosphere. So, the future of healthcare is in the Quantum computing which will help in arming the healthcare workers with super technologies, helping in super treatment protocols for the benefit of the patients and people across the globe.

Quantum computing will help in revolutionizing the medical world with the help of technologies which will lower the burden of uncertain treatments.

Not only this, like presently the humanity is suffering from the corona pandemic, and all of us are looking for treatment. If Quantum computing is put into practice we would be able to find out treatment not only for Corona but all be ready for any form of the pandemic which may occur in future. ${ }^{1}$

So, Quantum computing is basically a futuristic healthcare technology which will make the healthcare workers armed for dealing with any kind of uncertainty which may occur in present and near future. 
How will Quantum computing help in reshaping the healthcare Industry?

Well, Quantum computing works on the principle of Quantum mechanics. Now, Quantum mechanics is in discussion for many decades. It means that the computer powered by Quantum mechanics will empower it with revolutionizing speeds and accurate as well as precise calculations.

Now, how will it help in healthcare? It's a big discussion at present, and the situation is currently in its novice scenario. However, it is predictable that quantum computers will help in finding out a better treatment plan for the patients, after recording their data, such as age, food habits, other habits, history of any medication, as well as any history of past surgeries or treatments. Quantum computers can decode the best treatment plan for each specific patient who comes to the hospital or the doctor's clinic. Clinicians with the help of quantum computers can quickly analyze the situation of each respective patient and will help in better decision making for the appropriate treatment plan. This means, that Quantum computers will help in changing the current healthcare scenario to completely patient-centric or end user-centric.

Another advantage of Quantum computing is in Medical Diagnostic Industry, where it will empower the experts with precise and fine imaging of deep tissues, and help in finding the appropriate location of the damage inside the patient's body. ${ }^{2}$

Apart from this, Quantum computing will help researchers vastly through their quantum mechanics powered systems. How? Well, researchers take at a present a lot of time to find an appropriate drug candidate. Quantum computers can analyze big data in a very short span of time and will help in finding out better results for the whole research and development industry.

Healthcare at present is in a preventive scenario. When Quantum computers are used widely in the healthcare sector, the complete data of the person can be recorded, and it will take the form of Big Data. Now, from this huge big data, quantum computers will find out better preventive action plans for the clinicians as well as healthcare workers, so that real-time basis preventive therapeutic action plans can be built, and the same can be benefited to the end-user, i.e. the patient itself. 3

Quantum mechanics empowered computers, will revolutionize the whole healthcare industry, where faster and better treatment protocols will take shape in a real-time basis, avoiding the hassles faced by the patients for the disease for which cure is available and also for the ailments for which no cure is available at present.

The thing to be understood is that each patient's body is genetically different from the other patient. So, quantum computers can help in developing patient-centric healthcare technologies. Also, based on the same genetic makeup, and the same data available in the qubits form in computers, the quantum computer can with speeds calculate the preventive treatment action plans.

Thus, this is how, quantum computers will revolutionize the healthcare industry, from preventive to patient-centric and to predictive environment. 4 
As each person's genetic makeup is different, the quantum computers, and quickly calculate the future of health for the patient itself. This is how quantum computers will change the healthcare industry to a new phase i.e. PREDICTIVE HEALTHCARE TECHNOLOGIES.

\section{Conflict of Interest}

There is no conflict of interest while publishing this manuscript.

\section{Reference}

1. What can Quantum computing do to healthcare? [Online]. Dated: $31^{\text {st }}$ October, 2019[cited: 05 September 2020]. Available at: URL: https://medicalfuturist.com/quantumcomputing-in-healthcare/

2. Joshi, Naveen. How quantum computing can improve healthcare systems[Online]. Dated: November 27, 2019. [Cited: 09-092020]. Available at: URL: https://www.allerin.com/blog/how- quantum-computing-can-improvehealthcare-systems

3. Fontana, Carmen. Quantum computing is no longer a thing of science fiction. The technology is here, now. Healthcare is one industry that holds a lot of potential to integrate quantum.[Online]. Dated: January 18, 2020[cited 9 September, 2020]. Available at: URL: https://centricconsulting.com/blog/howquantum-computing-will-impacthealthcare/

4. Solenov, D, Breiler, J, et al. The Potential of Quantum Computing and Machine Learning to Advance Clinical Research and Change the Practice of Medicine. Mo Med. 2018 Sep-Oct; 115(5): 463-467. 